\title{
COMPLETELY REDUCIBLE OPERATORS
}

\section{PETER ROSENTHAL ${ }^{1}$}

1. Introduction. Let $H$ be a separable complex Hilbert space. A (closed) subspace of $H$ is nontrivial if it is different from $\{0\}$ and $H$. A bounded linear operator $A$ on $H$ is completely reducible if whenever $M$ is a reducing subspace of $A$ of dimension greater than 1, the operator $A \mid M$ has a nontrivial reducing subspace. The spectral theorem implies that every normal operator is completely reducible. If $H$ is finite dimensional then every completely reducible operator is normal. This is not the case in general, however, as the example given below shows.

Our main results are sufficient conditions that a completely reducible operator have a reducing eigenvector, i.e. an eigenvector that is also an eigenvector of the adjoint of the operator. For normal operators, of course, every eigenvector is a reducing eigenvector. Andô [1] has shown that a compact operator which has the property that every invariant subspace is reducing must be normal. Our techniques are similar to Andô's, and Corollary 3 below generalizes his result to the case of polynomially compact operators.

2. An example. Let $B$ denote the Hermitian operator consisting of multiplication by the independent variable on $L^{2}(0,1)$. P. R. Halmos has shown (unpublished) that every reducing subspace of the operator

$$
\left(\begin{array}{ll}
0 & B \\
0 & 0
\end{array}\right),
$$

acting on the space $L^{2}(0,1) \oplus L^{2}(0,1)$, is of the form $N \oplus N$, where $N$ is a reducing subspace of $B$. It follows that

$$
\left(\begin{array}{ll}
0 & B \\
0 & 0
\end{array}\right)
$$

is a completely reducible operator, although it is clearly not normal.

A slight modification of Halmos's example shows that a completely reducible operator can have a spanning set of eigenvectors

Received by the editors April 10, 1967.

1 The author is very grateful to Professor P. R. Halmos for his suggestions regarding the material in this paper and the preparation of the manuscript. 
and still be nonnormal. We show that every reducing subspace of the operator

$$
A=\left(\begin{array}{ll}
0 & B \\
0 & 1
\end{array}\right)
$$

is of the form $N \oplus N$, where $N$ reduces $B$. Our argument is essentially the same as Halmos's.

First note that

$$
A^{*}=\left(\begin{array}{ll}
0 & 0 \\
B & 1
\end{array}\right) \text { and } A^{*} A=\left(\begin{array}{cc}
0 & 0 \\
0 & B^{2}+1
\end{array}\right) .
$$

Now let $M$ be any reducing subspace of $A$. Let $N$ be the reducing subspace of $B$ generated by the set of vectors $f$ in $L^{2}(0,1)$ such that there exists a $g$ in $L^{2}(0,1)$ with $\langle f, g\rangle$ or $\langle g, f\rangle$ in $M$. Then clearly $M$ is contained in $N \oplus N$. To prove the reverse inclusion, let $\langle h, k\rangle$ be any element of $M$. Then the fact that $\left(A^{*} A\right)^{n}\langle h, k\rangle$ is in $M$ for every positive integer $n$ implies that $\{0\} \oplus \bigvee_{n=1}^{\infty}\left\{\left(B^{2}+1\right)^{n} k\right\}$ is contained in $M$. But polynomials without constant term in the variable $\left(x^{2}+1\right)$ are uniformly dense in the space of continuous functions on $[0,1]$. Therefore $\langle 0, k\rangle$ is in $M$, and then so is $\langle h, 0\rangle$. If we apply $A$ and $A^{*}$ to these vectors we see that $\{0\} \oplus \bigvee_{n=0}^{\infty}\left\{B^{2 n} k\right\},\{0\} \oplus \bigvee_{n=0}^{\infty}\left\{B^{2 n+1} h\right\}$, $\bigvee_{n=0}^{\infty}\left\{B^{2 n} h\right\} \oplus\{0\}$, and $\bigvee_{n=0}^{\infty}\left\{B^{2 n+1} k\right\} \oplus\{0\}$ are each contained in $M$.

Now if $\left\langle f_{1}, f_{2}\right\rangle$ is in $N \oplus N$ then the foregoing shows us how to prove that $\left\langle f_{1}, 0\right\rangle$ and $\left\langle 0, f_{2}\right\rangle$ are in $M$. Hence $N \oplus N$ is contained in $M$.

3. Results. The above example shows that a completely reducible operator need not have a reducing eigenvector even if it has a spanning set of eigenvectors and is polynomially compact; $\left(A^{2}-A=0\right.$ in the example). Our results show that a slight strengthening of either of these conditions does imply that a completely reducible operator has a reducing eigenvector.

Lemma. If $\left\{M_{\alpha}\right\}$ is a totally ordered family of subspaces of a separable Hilbert space, and if $N=\bigcap_{\alpha} M_{\alpha}$, then there is a countable subfamily $\left\{M_{\alpha_{i}}\right\}$ such that $N=\bigcap_{i} M_{\alpha_{i}}$ and $M_{\alpha_{i+1}} \subset M_{\alpha_{i}}$ for each $i$.

Proof. ${ }^{2}$ Taking set-theoretic complements, we get $N^{\prime}=\mathrm{U}_{\alpha} M_{\alpha}^{\prime}$. Since $N^{\prime}$ has the Lindelö property, there is a countable subcover $\left\{M_{\alpha_{i}}^{\prime}\right\}$. Then $N=\bigcap_{i} M_{\alpha_{i}}$. To get $M_{\alpha_{i+1}} \subset M_{\alpha_{i}}$ we simply discard $M_{\alpha_{i+1}}$ if $M_{\alpha_{i+1}} \nsubseteq M_{\alpha_{i}}$.

Theorem 1. If $A$ is completely reducible, and if the subspace $E_{\lambda}$ $=\{x: A x=\lambda x\}$ is finite dimensional, then $E_{\lambda}$ reduces $A$.

2 This proof is due to C. R. MacCluer. 
Proof. Let $\mathcal{F}$ denote the family of subspaces of $H$ that reduce $A$ and meet $E_{\lambda}$ in a subspace other than $\{0\}$. By the Hausdorff maximal principle there is a maximal chain $\left\{M_{\alpha}\right\}$ in $\mathcal{F}$. If we let $N$ be $\bigcap_{\alpha} M_{\alpha}$, then $N$ clearly reduces $A$. We will show that $N$ is in $\mathcal{F}$ and that $N$ is one dimensional.

Choose a countable subfamily of $\left\{M_{\alpha}\right\}$ by the Lemma. Then for each $i$ choose an $x_{i}$ in $M_{\alpha_{i}}$ such that $\left\|x_{i}\right\|=1$ and $A x_{i}=\lambda x_{i}$. Some subsequence of $\left\{x_{i}\right\}$ converges, to some $x$, since $E_{\lambda}$ is finite dimensional. Then $x$ is in $N$, since the sequence $\left\{x_{i}\right\}$ is eventually in each $M_{\alpha_{i}}$. Also $\|x\|=1$ and $A x=\lambda x$. Thus $N$ is in $\mathcal{F}$ and the dimension of $N$ is at least 1.

If the dimension of $N$ were greater than 1 then $A \mid N$ would have a nontrivial reducing subspace $L$. Then at least one of $L$ and $L^{\perp} \cap N$ would be in $\mathcal{F}$, contradicting the fact that $N$ is the intersection of the subspaces in a maximal chain in $\mathcal{F}$.

Thus the dimension of $N$ is 1 and $N$ contains a reducing eigenvector $x$ in $E_{\lambda}$. If the dimension of $E_{\lambda}$ is greater than 1 we can apply the above proof to $A \mid\{x\}^{\perp}$ and get another reducing eigenvector in $E_{\lambda}$. If we repeat this process finitely many times we will get an orthonormal basis for $E_{\lambda}$ consisting of reducing eigenvectors.

CoROllary 1. If $A$ is completely reducible and has a spanning set of eigenvectors each of which corresponds to an eigenvalue of finite multiplicity, then $A$ is normal.

Proof. Each eigenvector in the spanning set is reducing, by Theorem 1, and thus $A$ can be written as a diagonal matrix.

THEOREM 2. If $A$ is completely reducible, and if there exists a nonzero compact operator $T$ such that every reducing subspace of $A$ reduces $T$, then $A$ has a reducing eigenvector.

Proof. The proof is similar to the proof of Theorem 1 . Here we let $\mathcal{F}$ denote the family of subspaces $M$ that reduce $A$ and that have the property that $\|T \mid M\|=\|T\|$. Let $N$ be the intersection of the subspaces in a maximal chain in $\mathcal{F}$ and write $N=\bigcap_{i} M_{\alpha_{i}}$ by the Lemma. We will be done if we show that the dimension of $N$ is 1 .

A compact operator attains its norm; thus for each $i$ there is an $x_{i}$ in $M_{\alpha_{i}}$ such that $\left\|x_{i}\right\|=1$ and $\left\|T x_{i}\right\|=\|T\|$. Choose a subsequence of $\left\{x_{i}\right\}$ that converges weakly to some $x$. Then $\|T x\|=\|T\|$ and thus $\|x\|=1$. Also, $x$ is in $N$ since $\left\{x_{i}\right\}$ is eventually in each $M_{\alpha_{i}}$. Thus $N$ is in $\mathcal{F}$. If the dimension of $N$ were greater than 1 there would be a nontrivial reducing subspace $L$ of $A$ properly contained in $N$. But the fact that $T$ attains its norm on $N$ implies that $T$ attains its norm on $L$ 
or on $L^{\perp} \cap N$. Thus at least one of $L$ and $L^{\perp} \cap N$ is in $\mathcal{F}$, contradicting the definition of $N$.

CoRollary 2. A compact completely reducible operator is normal.

Proof. Let $A$ be compact and completely reducible, and let $\varepsilon$ be a maximal orthonormal set of reducing eigenvectors of $A$. It suffices to show that $\mathcal{E}^{\perp}$ is $\{0\}$. If $\mathcal{E}^{\perp}$ is not $\{0\}$ then either $A \mid \mathcal{E}^{\perp}=0$ or Theorem 2 applies, with $T=A$. In either case the maximality of $\mathcal{E}$ is contradicted.

Corollary 3. If $A$ is polynomially compact, (i.e. there is a nonzero polynomial $p$ such that $p(A)$ is compact), and if every invariant subspace of $A$ is reducing, then $A$ is normal.

Proof. First, such an operator is completely reducible, by the Bernstein-Robinson invariant subspace theorem for polynomially compact operators [2], [3]. Let $\varepsilon$ be a maximal orthonormal set of reducing eigenvectors of $A$. If $\varepsilon^{\perp} \neq\{0\}$, consider $A \mid \varepsilon^{\perp}$. If $p(A) \mid \mathcal{E}^{\perp}$ is not 0 then $A$ has a reducing eigenvector in $\varepsilon^{\perp}$ by Theorem 2 , with $T=p(A)$. If $p(A) \mid \mathcal{E}^{\perp}$ is 0 then $A \mid \mathcal{E}^{\perp}$ has an eigenvector by the spectral mapping theorem. But every eigenvector of $A$ is reducing by hypothesis.

Corollary 4. If $A$ is completely reducible, and if $p(A)$ is compact and has finite-dimensional nullspace for some polynomial $p$, then $A$ is normal.

Proof. As in the previous corollaries, let $\varepsilon$ be a maximal orthonormal set of reducing eigenvectors of $A$. If $\varepsilon^{\perp}$ is finite dimensional then $A \mid \mathcal{E}^{\perp}$ is normal and the proof is finished. If $\mathcal{E}^{\perp}$ is infinite dimensional then $p(A) \mid \mathcal{E}^{\perp}$ is not 0 and Theorem 2 applies.

CoROllary 5. If the von Neumann algebra generated by a completely reducible operator $A$ contains a nonzero compact operator, then $A$ has point spectrum.

Proof. The hypotheses imply that $A$ and the compact operator satisfy the conditions of Theorem 2 .

THEOREM 3. Let $A$ and $B$ be operators on $H$ with disjoint spectra. Then an operator $S$ commutes with $A \oplus B$ on $H \oplus H$ if and only if $S=C \oplus D$, where $C$ commutes with $A$ and $D$ commutes with $B$.

Proof. Let

$$
S=\left(\begin{array}{ll}
C & E \\
F & D
\end{array}\right)
$$


commute with $A \oplus B$. We must show that $E=0$ and $F=0$. Now

$$
S(A \oplus B)=\left(\begin{array}{ll}
C A & E B \\
F A & D B
\end{array}\right) \text { and }(A \oplus B) S=\left(\begin{array}{ll}
A C & A E \\
B F & B D
\end{array}\right) .
$$

Therefore $A E=E B$ and $B F=F A$. A theorem of Rosenblum [4] implies that these equations can hold only if $E=0$ and $F=0$ (because of the fact that the spectra of $A$ and $B$ are disjoint).

Corollary 6. If $A$ and $B$ are completely reducible and have disjoint spectra then $A \oplus B$ is completely reducible.

Proof. It follows from Theorem 3 that every projection that commutes with $A \oplus B$ is of the form $P \oplus Q$. Thus every reducing subspace of $A \oplus B$ is of the form $M \oplus N$, where $M$ reduces $A$ and $N$ reduces $B$.

4. Remarks. The above theorems and corollaries all hold for operators on nonseparable spaces too; this follows from the fact that every operator can be written as the direct sum of operators on separable spaces.

It would be interesting to find other sufficient conditions that a completely reducible operator be normal. In particular, if $A$ is completely reducible and every invariant subspace of $A$ is reducing must $A$ be normal?

AdDED IN PROOF. The case of Corollary 3 where $p(z)=z^{n}$ has been provén by T. Saitô in Some remarks to Ando's Theorems, Tôhoku Math. J. (2) 18 (1966).

\section{REFERENCES}

1. T. Andô, $A$ note on invariant subspaces of a compact normal operator, Arch. Math. 14 (1963), 337-340.

2. A. R. Bernstein and A. Robinson, Solution of an invariant subspace problem of K. T. Smith and P. R. Halmos, Pacific J. Math. 16 (1966), 421-431.

3. P. R. Halmos, Invariant subspaces of polynomially compact operators, Pacific J. Math. 16 (1966), 433-437.

4. M. Rosenblum, On the operator equation $B X-X A=Q$, Duke Math. J. 23 (1956), 263-269.

The University of Michigan and

The University of Toronto 